\title{
Téoros
}

Revue de recherche en tourisme

\section{L'aménagement des fronts de mer urbains} Politiques contradictoires à Québec et à Montréal

\section{Léonce Naud}

Volume 6, numéro 2, juillet 1987

Le Saint-Laurent magnétique

URI : https://id.erudit.org/iderudit/1080512ar

DOI : https://doi.org/10.7202/1080512ar

Aller au sommaire du numéro

Éditeur(s)

Université du Québec à Montréal

ISSN

0712-8657 (imprimé)

1923-2705 (numérique)

Découvrir la revue

Citer cet article

Naud, L. (1987). L’aménagement des fronts de mer urbains : politiques contradictoires à Québec et à Montréal. Téoros, 6(2), 35-36.

https://doi.org/10.7202/1080512ar d'utilisation que vous pouvez consulter en ligne.

https://apropos.erudit.org/fr/usagers/politique-dutilisation/ 


\title{
Politiques contradictoires à Québec et à Montréal L'aménagement des fronts de mer urbains
}

\author{
Par Léonce Naud*
}

Le développement du nautisme, la multiplication des croisières et des activités recreatives reliées aux plans d'eau suscitent un intérềt accru envers l'aménagement optimal des berges urbaines. surtout lorsqu'elles donnent sur des cours d'eau de l'importance du Saint-Laurent. Un aménagement en bordure d'un canal, d'une rivière ou d'un fleuve peut améliorer grandement la qualité de vie pour un grand nombre mentionnons. l'exemple bien connu du canal Rideau à Ottawa = ou encore résulter en un désastre de béton, comme dans le cas de la "solution finale" apportée à la rivière Saint-Charles à Québec. Qui plus est, on se rend compte de plus en plus qu'une réjuvénation réussie de zones urbaines riveraines peut provoquer d'importantes retombes touristiques et kconomiques. Par ailleurs, plus le cours d'eau est en général vaste, majestueux et dépollué, plus il suscitera la convoitise publique... et privéc. Il est notoire que du point de vue de l'inté" rêt public, la plupart de ces plans grandioses finissent plutot mal.

Au Québec, le gouvernement fédéral, fort de nombreux terrains excédentaires résultant du déplacement des activités dans les ports de Montréal et de Québec, présenta vers 1974 d'ambitieux projets de réamenagement urbain: les rapports URBEX. Le ministère fédéral des Affaires urbaines aboli depuis - y indiquait sans détour son intention d'assumer le leadership en matière d'aménagement urbain, domaine jusqu'alors de juridiction provinciale. Plus précisément, en mettant de l'avant des projets expérimentaux d'aménagement urbain en d'anciennes zones industrialoportuaires, Ottawa entendait indiquer la voie à suivre notamment au ministere québécois des Affaires municipales. Tel que le déclara crüment l'architecte principal des projets URBEX, " "... Il faut une certaine attitude mentale et une détemination qu'on ne retrouve malheureusement pas à Québec" (11)"

\footnotetext{
* Leonce Naud est gégraphe au Secrétariat à la misk en valeur du Saint-Laurent; le 7 janvier 1907; il a déposed, a titre personnel, un rapport de 245 pages rechamant un moratoire dans is Veux-Port de Qubbec. Los plans fodbraux de 1974
}

Dans le cas de Montréal, le projet URBEX, qui suggerrait une pléthore de constructions en bordure du fleuve, fut rapidement mis de cơté, notamment lorsque les autorités municipales firent remarquer qu'on créerait ainsi une barriere entre le VieuxMontréal et le Saint-Laurent. La Ville fit savoir qu'elle favorisait plutốt la mise en place d'un parce doté d'aménagements légers et qu'elle ne voulait pas voir de bátiments à cet endroit. Dans le cas de Qué$\mathrm{bec}_{+}$la surprise fut totale: personne n'était au courant au gouvernement du Québec et Ottawa précisait même qu'il avait décidé de completer le concept avant de consulter les partenaires. Reçu fraichement en 1974 , le plan entra alors dans une période de latence, pour refaire soudain surface quatre ans plus tard, en février 1978, en plein Carnaval, à l'aube d'une campagne électorale fédérale.

\section{Montréal et Québec divergent}

À Montréal, la question de l'aménagement du "front de mer" se transforma bientôt en véritable saga et provoqua une participation du public absolument remarquable. Une confrontation s'étendant sur dix ans mit aux prises d'une part les autorités fédérales et les firmes dont elles avaient retenu les services, d'autre part la grande majorité des citoyens consultés, des architectes et urbanistes indépendants ainsi que les autorités municipales. La controverse: Ottawa préconisait des immeubles à bureaux, une foire commerciale, des habitations de luxe, le tout sur fond de privatisation des lieux; I'autre partie voulait garder le lieu public, rejetait les vocations

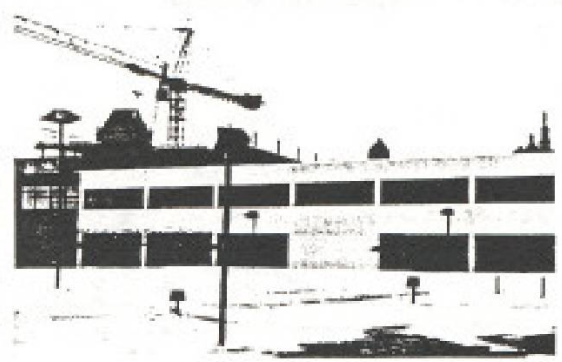

Vue vers Qubbec, Vilie du Patrimoine mondial, un mur continu sépare desormais la ville du fleuve. résidentielles, d'édifices à bureaux, de commerces, favorisait les espaces verts et dégagés ainsi que l'accessibilité physique et visuelle. Cette dernière option finit par l'emporter et les plans actuellement retenus reflètent assez fidèlement les recommandations d'une vaste opération de consultations publiques tenue l'an dernier dans la métropole.

Le hic, c'est que durant la méme période (1977-1987) les mêmes autorités fédérales qui consultaient a tour de bras a Montreal se comportaient au bord du fleuve à Québec en véritables satrapes et construisirent a toute vitesse, sans consultation aucune, un mur solide de verre, d'acier et d'aluminium entre la Vieille-Ville et le fleuve.

\section{Un comportement inexpliqué}

Il faut préciser ici que, durant sa période d'hibernation (1974-1978), le plan URBEX pour le "vieux port" de Québec subit maintes transformations. Vers 1977, le concept d'aménagement élaborế par Parcs Canada mettait de l'avant un concept de parc public qui maintenait à l'intérieur de limites "raisonnables" le remblaiement du fleuve. Deux ans plus tard, en 1979, un nouveau plan, élaboré cette fois par la firme Pluram, prévoyait un plus grand empiètement sur le fleuve ainsi qu'un plus grand nombre de constructions, mais y conservait quand meme le "parc de la pointe à Carcy $^{* *}$. Mais c'est véritablement au cours de 1'hiver 1980-1981 que le mythe du "quartier urbain modele" reprit soudain toute sa force, et que le projet initial devint, en fait, "tun autre projet" ${ }^{\text {". Pourquoi? }}$

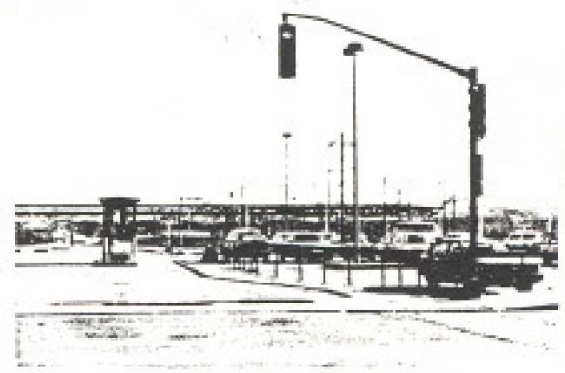

Vue vers le fleuve a Montráal, photo prise de la rue Dalhousie. 130 millions de dollars plus tard, on ne voit pas le fleuve. 
A l'automne 1980, le premier ministre du Québec, M. René Lévesque, avait annoncé la décision du gouvernement du Québec d'investir massivement à la limire même des propriétés fédérales, rue Dalhousie, 25 millions pour 1'Université du Québec et 35 millions pour un futur Musée national de la civilisation. Soit dit en passant, ce Musée devait "s'ouvrir largement sur le fleuve et les civilisations atlantiques". C'est apparamment cette décision qui précipita le fédéral dans une frénésie immobilière en bordure du fleuve - entre le Fleuve et la Ville -, qui visait à créer un environnement canadian tellement hightech, tellement hyper, que le visiteur serait saisi, étonné, estomaqué par une expérience inoubliable; serait en un mot "conquis" par l'aspect surprenant, nouveau, inattendu et étranger de ce nouvel environnement urbain. (À l'époque, rappelons-nous, chaque gouvernement mettait toute son ardeur à conquérir "les esprits et les coeurs", car il $\mathrm{y}$ avait un référendum qui pointait à 1'horizon).

Cette option - aux motifs fondamentalement politiques - n'a jamais fait l'objet de débats ni de consultations publiques, et pour cause: les mêmes décideurs fédéraux qui construisaient à tour de bras en face de Québec procédaient en mëme temps à des consultations et à divers sondages du public montréalais sur le Vieux-Port à cet endroit. Ces consultations démontraient que la population en général de la métropole rejetait carrément condominiums de luxe, bureaux, commerces, hótels ainsi que toute idée de privatisation de l'espace. Ces décideurs politiques fédéraux se sont bien gardés de le dire à Québec! Néanmoins, les citoyens de Québec qui ont pu exprimer leur avis ont dit la mëme chose que les Montréalais. C'est ainsi que la Société du Vieux-Port de Québec a violé pratiquement tous les concepts et approches d'aménagement finalement retenus dans la métropole pour l'aménagement du Vieux-Port de Montréal.

Actuellement, on poursuit à Québec l'option d'aménagement décidée au cours de l'hiver 1980-1981, à savoir l'urbanisation extensive - dans un style architectural apatride - de terrains acquis à même le fleuve, au détriment du contact de la Vieille-Ville avec ce cours d'eau majestueux et au prix de l'obstruction quasi-totale de la vue du fleuve vers la ville et viceversa. L'approche d'aménagement est similaire à celle qui a fini par être rejetée à Montréal, notamment parce qu'elle signifiait en clair l'accaparement du site par une poignée de nantis au détriment de l'ensemble de la population. Notons que l'option d'une urbanisation et d'un "développement ${ }^{* *}$ extensif du rivage urbain vient ćgalement d'être rejetté à Toronto où les opérations de la Société fédérale Harborfront sont actuellement "gelées".

En résumé, on continue à Québec de faire exactement le contraire de tout ce qu'on prétend avoir du bons sens à Montréal et à Toronto.

\section{Le "quartier urbain modèle"}

Le mythe fondamental qui sous-tend toute la "philosophie urbaine" et les réalisations du gouvernement fédéral dans cette ancienne partie du port de Québec est la croyance qu'il faut y ériger un " "quartier urbain modèle*". Comme tous les mythes, celui-ci fait surface dans les grandes occasions, les grandes cérémonies ou les grandes justifications. Quelqu 'un critique? On répondra: "Mais on est simplement d'avant-garde... On construit pour l'an 2,000 !" Même en 1974, il fallait une bonne dose d'arrogance pour arriver d'Ottawa, s'installer au bord du fleuve en face de Québec et déclarer ni plus ni moins: "Assurément, cette ville a besoin d'un quartier urbain modele, un quartier d'avant-garde, digne de servir d'exemple et qui sera la voie de l'avenir...". Depuis, l'entreprise est devenue simplement grotesque, car c'est la ville elle-méme - plus précisément ce Vieux-Québec qu'on a tant boudé et méprisé -, qui a êté déclarée ni plus ni moins ville urbaine modèle par l'UNESCO. Le monde entier la découvre graduellement, gråce au travail accompli par l'Hôtel de Ville, par la province. par Parcs Canada, par l'UNESCO, etc., et également par tant de citoyens qui, désormais, rénovent avec amour leurs vieilles pierres et leur fer forgé et attirent des milliers de visiteurs, Québécois el étrangers, sans parler de nouveaux résidents.
Pendant ce temps, abritée derrière ses remparts d'aluminium, de verre et de poutrelles de mécano qui empechent de voir le fleuve, habituée à un fric inépuisable provenant des contribuables, la Société du Vieux-Port de Québec continue obstinément à bătir son "quartier urbain modèle" et $\pi^{\prime} a$ encore rien appris. (Quand on y pense, depuis que Québec est consacrée Ville du patrimoine mondial, l'aluminium. le verre et les tuyaux criards font plutót passé....).

\section{Un dossier en or}

En guise de conclusion, signalons qu'il existe peu de dossiers urbains qui recèlent autant d'imprévus et de découvertes potentielles que "l'histoire immédiate" de ces 130 millions de dollars qui ont été engloutis en quelques années, sans aucune consultation publique, dans des battisses inutiles et nuisibles, situées entre Québec et le fleuve, constructions qui restent d'ailleurs inoccupées jusqu'à ce jour. Les éléments d'analyse ci-haut résultent d'une recherche rapide, pressée par les évếnements. Le sujet s'avère un véritable trésor pour du "journalisme d'investigation" " ou encore pour des universitaires spécialisés en aménagement, urbanisme ou géographie urbaine. L'auteur espère susciter ainsi quelques vocations et quelques thèses... d'interet immédiat, et sera heureux de mettre à la disposition des intéressés toute la documentation dont il dispose. $f$
Retifinence

(1) Le Devoir, ife 19 avril 1974.

\section{Vue de coupe de l'esplanade actuelle}

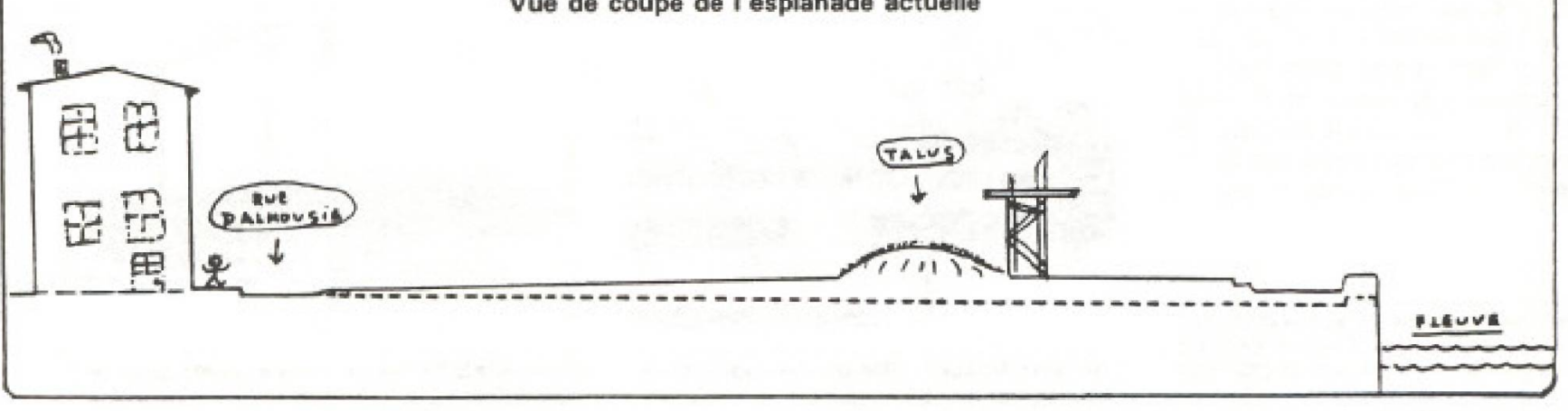

La ligne en pointille indique comment l'esplanade eut dũ âtre faite pour qu'on voie le fleuve de la ville. 